\title{
Barrère Céline et Rozenholc Caroline, Les lieux de mobilité en question. Acteurs, enjeux, formes, situations
}

Hadrien Dubucs

\section{(2) OpenEdition}

Édition électronique

URL : https://journals.openedition.org/remi/13984

DOI : 10.4000/remi.13984

ISSN : $1777-5418$

Éditeur

Université de Poitiers

\section{Édition imprimée}

Date de publication : 1 décembre 2019

Pagination : 301-303

ISBN : 979-10-90426-65-8

ISSN : 0765-0752

Référence électronique

Hadrien Dubucs, «Barrère Céline et Rozenholc Caroline, Les lieux de mobilité en question. Acteurs, enjeux, formes, situations ", Revue européenne des migrations internationales [En ligne], vol. 35 - n³ et 4 । 2019, mis en ligne le 01 décembre 2019, consulté le 15 avril 2022. URL : http:// journals.openedition.org/remi/13984; DOI : https://doi.org/10.4000/remi.13984

Ce document a été généré automatiquement le 15 avril 2022.

(c) Université de Poitiers 


\title{
Barrère Céline et Rozenholc Caroline, Les lieux de mobilité en question. Acteurs, enjeux, formes, situations
}

\author{
Hadrien Dubucs
}

\section{RÉFÉRENCE}

Barrère Céline et Rozenholc Caroline (2018) Les lieux de mobilité en question. Acteurs, enjeux, formes, situations, Paris, Karthala, 186 p., ISBN : 978-2-8111-1905-8

1 Au sein d'une production scientifique francophone très abondante sur la question des mobilités spatiales, l'ouvrage dirigé par Céline Barrère et Caroline Rozenholc se distingue à plusieurs titres. Il restitue une journée d'études tenue en janvier 2016 dans le cadre du Collège international des sciences du territoire, mais ne se limite pas à la publication d'actes. En effet, les sept contributions, résolument empiriques et très variées en termes de terrain d'étude et d'approche disciplinaire, sont mises en perspective sur un plan théorique par une introduction très dense. Celle-ci montre bien toute la difficulté conceptuelle - liée au caractère flottant de la notion même de lieu mais aussi le caractère très heuristique de questionner la fabrication et la transformation des lieux par le prisme des mobilités, et réciproquement de comprendre les mobilités grâce à une approche localisée, voire micro-localisée. Celle-ci permet en particulier d'enrichir l'analyse des mobilités spatiales bien au-delà de leurs facteurs et enjeux techniques, économiques et politiques, en intégrant notamment ses dimensions culturelles et symboliques. En effet, les textes rassemblés dans l'ouvrage proposent des analyses qui ont trait au mémoriel, au symbolique, ou encore aux processus d'identification. La richesse de l'ouvrage tient aussi à la variété des objets comme des approches disciplinaires et méthodologiques développées dans les différents chapitres. Certains sont centrés sur des groupes d'individus mobiles 
(migrants sahraouis du Bordelais, volontaires catholiques français en Asie et dans l'océan Indien), d'autres sur des lieux (la petite ville de Sartène en Corse, une ville nouvelle en banlieue de Shanghaï, un vallon des Pyrénées espagnoles). Un autre apport certain de l'ouvrage tient à la variété des formes et échelles de mobilités prises en considération pour analyser les lieux: processions religieuses, migrations professionnelles, ou encore cheminements touristiques. Mais plus qu'une juxtaposition d'études de cas abordant des types de mobilités distincts, Les lieux de mobilité en question permet de mieux comprendre comment s'articulent plusieurs formes de mobilités spatiales, selon un horizon théorique désormais bien établi en sciences sociales anglophones et francophones dans le sillage de ce que certains auteurs qualifient de «mobility turn » (Faist, 2013). C'est donc un réel intérêt de l'ouvrage que de proposer plusieurs études empiriques qui assument clairement, bien que parfois non explicitement, cette ambition théorique. Le texte de Pierre-Yves Trouillet («Mobilité et temples hindous en diaspora ») par exemple apporte une contribution passionnante à la compréhension des relations possibles entre une mobilité de grande ampleur, en l'occurrence la diaspora hindoue des Tamouls d'Inde et de Sri Lanka, construite sur plus d'un siècle et connectant des régions très éloignées les unes des autres (du Canada à l'Australie), et des mobilités qui se jouent à l'échelle micro-locale (celle de l'espace du temple) et selon des pratiques du quotidien (déambulations rituelles). Des pistes intéressantes sont ouvertes pour dépasser la simple juxtaposition des mobilités spatiales pour une population considérée, et identifier la façon dont elles interagissent. Ainsi, l'agencement et la disposition des objets de culte à l'intérieur et autour du temple sont conçus pour faire écho à l'histoire diasporique et aux lieux qu'elle met en jeu : la mobilité micro-locale individuelle des fidèles se comprend alors comme une forme de narration d'une mobilité collective, historique et d'échelle mondiale. Cette combinaison étroite du rituel et du mémoriel illustre de manière frappante comment un lieu peut être «de mobilités » au pluriel, et engage les chercheurs à étudier ce lieu sans se limiter aux formes les plus immédiatement visibles de déplacements qui s'y déploient.

2 Cette observation, qui traverse plusieurs des contributions rassemblées dans l'ouvrage, engage également une réflexion méthodologique : comment saisir les mobilités en tant qu'elles interagissent avec un lieu et ses caractéristiques fixes - du moins spatialement, car elles peuvent évoluer dans le temps comme le souligne avec force l'introduction de Céline Barrère et Caroline Rozenholc où les lieux sont définis comme "des reconfigurations des temps et des espaces ». La question des méthodes n'est pas directement abordée en tant que problématique transversale et on peut le regretter tant elle est intrinsèquement liée à la difficulté à laquelle se confrontent les chercheurs abordant cette dialectique du fixe et du mouvant qui caractérise les lieux de mobilité. Néanmoins, les différentes contributions décrivent avec précision les approches et les méthodes mobilisées, qui frappent par leur diversité. Ainsi, l'étude par Pierre-Louis Ballot et Emma-Sophie Mouret de la «construction patrimoniale» de la Route Nationale 7 combine les méthodes de l'analyse historique - documents d'archives écrites, photographiques et audiovisuelles - et de l'analyse géographique - entretiens avec des acteurs contemporains de la mise en tourisme et de l'action territoriale. L'observation ethnographique tient également une place décisive dans l'étude de lieux d'innovation politique (Beneficio, dans le chapitre de Marion Kameneff) ou de fête religieuse (étude du Catinacciu à Sartène par Davia Benedetti). Quant à l'enquête qualitative par entretiens approfondis, elle permet à Martin Minost de comprendre les 
choix résidentiels et les rapports à leur lieu de vie qu'entretiennent les habitants du quartier "anglais» de Thames Town, au cœur d'une ville nouvelle en banlieue de Shanghai.

3 Le matériau visuel occupe une place importante dans l'appréhension des relations entre lieux et mobilités. Plusieurs des chapitres sont illustrés par des photographies en couleurs - effort éditorial qu'il convient de saluer - qui, par leur diversité, montrent bien tout le défi consistant à fixer en image la manière dont des lieux sont produits par des mouvements, qui peuvent être saisis en direct, mais aussi sous la forme de traces matérielles de mobilités passées. Les espaces domestiques, par leur décor, leurs agencements et leurs caractéristiques matérielles, disent ainsi beaucoup des logiques d'occupation de leurs habitants parfois contraints à des formes de présence très transitoires (squats) ou durablement installés mais mobilisant des univers culturels et des références symboliques lointaines (dans le cas du quartier de la banlieue de Shanghai affichant sa «britannité »). D'autres photographies rendent compte de moments de mobilité collective (processions, déambulations) tout en illustrant les limites de l'image fixe pour rendre compte du lieu comme « spectacle vivant et scène de mobilités ». Cette expression évocatrice est développée par Christophe Imbert dans un chapitre conclusif de l'ouvrage qui s'attache à déployer certains des grands enjeux théoriques, mais aussi politiques et éthiques - à travers une discussion du "piège de l'enracinement » notamment - qui émergent dès lors que l'on considère le mouvement comme le principe même de ce qui fait et transforme un lieu. Au-delà de la dimension méthodologique de l'image, la question de la visibilité localisée des flux parcourt tout l'ouvrage et fait écho à d'autres champs de réflexion sur ce thème, notamment dans le domaine artistique. On peut ainsi citer l'exposition Mobile/Immobile tenue aux Archives Nationales au printemps $2019^{1}$ qui montre bien comment les démarches esthétiques et scientifiques se combinent utilement pour travailler cette question. La fécondité de ces échanges aurait sans doute gagné à être évoquée dans le passage en revue théorique proposé en introduction de l'ouvrage, résolument pluridisciplinaire (philosophie, anthropologie, géographie culturelle). On peut également mentionner que les références bibliographiques mobilisées par les auteurs intègrent relativement peu de travaux contemporains de sciences sociales anglophones qui abordent directement la question des lieux de mobilité .

\section{BIBLIOGRAPHIE}

Carpio Genevieve (2019) Collisions at the Crossroads: How Place and Mobility Make Race, Berkeley, University of California Press, 392 p.

Faist Thomas (2013) The mobility turn: a new paradigm for the social sciences?, Ethnic and Racial Studies, 36 (11), pp. 1637-1646.

Moores Shaun (2012) Media, Place and Mobility, London, Palgrave Macmillan, 144 p. 


\section{NOTES}

1. http://www.archives-nationales.culture.gouv.fr/mobile-immobile

2. À titre d'exemple on peut citer l'ouvrage récent (postérieur à l'ouvrage présenté) de l'historienne Carpio (2019) ou celui plus ancien de Moores (2012).

\section{AUTEURS}

\section{HADRIEN DUBUCS}

Géographe, Maître de conférences, ENeC, UMR 8185 CNRS-Paris IV Sorbonne 